\title{
Development of a new Q-meter module
}

\author{
A. Berlin, J. Herick*, W. Meyer, G. Reicherz \\ Experimentalphysik I, Ruhr-Universität Bochum \\ E-mail: jonas.herickerub.de
}

In the research field of polarized target physics the Q-meter is a well established technique to determine the polarization of solid target materials. One of the most common Q-meter modules is represented by the so called Liverpool-NMR, which was originally developed for the EMC experiment in the 1970s. However, since the module was built nearly 40 years ago, many components are no longer available. Thus the repair of existing modules and reproduction of new modules is often impossible.

Starting from that point, a new Q-meter module was developed and investigated for the use in polarized target experiments. The recent version was successfully used at A2 - Experiment at MAMI in Mainz.

XVIth International Workshop in Polarized Sources, Targets, and Polarimetry, PSTP2015,

14-18 September 2015,

Bochum, Germany

${ }^{*}$ Speaker. 


\section{Introduction}

For the operation and evaluation of measurement in particle physics experiments with polarized solid targets a precise knowledge of the sample polarization $P_{t}$ is essential. This can already be seen by the so-called figure of merit

$$
F O M \propto f^{2} P_{t}^{2} \propto \frac{1}{t_{\text {meas }}}
$$

of a polarized solid target, which characterizes the quality of the used target material with a dilution factor $f^{1}$ and is anti-proportional to the required measurement time $t_{\text {meas. }}$ for a given precision of these measurements.

In general, for the detection of target polarization the method of nuclear magnetic resonance is used. By means of a resonant circuit whose inductance is located in proximity to the sample, an alternating field is generated, which is oriented orthogonally to the external magnetic field $B_{0}$. If the frequency of the alternating field matches the Larmor frequency $\omega_{L}$ of the investigated spin system with the gyromagnetic factor $\gamma$,

$$
\omega=\omega_{L}=\gamma B_{0}
$$

transitions between the Zeeman levels are induced. However, this interaction only occurs for a narrow frequency range in the region of the Larmor frequency of the nuclei. Therefore, this effect is also called resonance absorption. Macroscopically, this results in a change of the sample magnetization, which is caused by the different occupied Zeeman levels of the sample.

Based on this change in magnetization, the NMR spectrum of the investigated sample can be determined. The area $A$ under the NMR line is proportional to the target polarization $P_{t}=k \cdot A$, with the so called enhancement factor ${ }^{2} k$.

\subsection{NMR methods}

In principle, there are two different methods for the detection of the NMR spectra of a sample. For the so-called pulsed NMR method, which is the current standard in many scientific fields, the sample magnetization is deflected from its original position parallel to the external field $B_{0}$ by a short and powerful RF-pulse. The subsequent relaxation of the magnetization back to equilibrium can be described by means of the Bloch equations [1,2], leading to a voltage induction into the NMR coil. This time-resolved voltage signal is commonly known as free induction decay signal FID and can be converted by a Fourier transformation into the desired NMR spectrum. However, for the exact determination of polarization by this method there arise different problems, which are attributable to a non-uniform signal excitation and a signal loss at the beginning of the FID detection.

Therefore, the well established continuous wave NMR technique is still the method of choice for

\footnotetext{
${ }^{1}$ The ratio of polarizable nuclei to the total number of nuclei in the target material.

${ }^{2}$ This factor is usually determined by a calibration in thermal equilibrium, because the corresponding polarization $P_{t, T E}$ can be calculated for a given magnetic field and a given temperature, so that a simple assignment $k=P_{t, T E} / A_{T E}$ can be made. This method is also called TE-calibration.
} 
the accurate detection of the undistorted NMR spectra and so for the measurement of the target polarization. Usually, this method is preformed at constant external field ${ }^{3} B_{0}$, while the frequency of the applied radio-frequency alternating field is swept over the resonance. Depending on the number and direction of the excited transitions between the Zeeman levels of the spin system, the power consumption or output of the resonant circuit changes. Since also the quality of the resonant circuit is affected, this set-up is also called Q-meter. The change of the quality of the resonant circuit can be detected as a variation of the radio-frequency power reflected from the oscillating circuit. However, by this means there are two difficulties. On the one hand, radio-frequency signals are difficult to handle, on the other hand, the signal to detect consists of two signal parts and is complex. Only the area below the imaginary part of the signal, the so-called absorptive signal component, is proportional to the target polarization $P_{t}$. To determine the amplitude of the radio-frequency signal one uses a diode detector followed by a low-pass filter which converts the RF-signal to a DC-signal proportional to the RF-amplitude. For the detection of the absorptive signal component, a phasesensitive detector is used, which is in phase with the exiting signal.

A system that works on this principle and has proven itself over the years in a variety of experiments is given by the so-called Liverpool module [3]. This was originally developed for the EMC experiment at CERN. Because of the relatively advanced age some components, particularly numerous radio-frequency amplifiers, are no longer available and it is complicated to repair an existing systems or to build up a new module.

\section{Developement of a new cw-NMR-module}

Therefore, the requirement for a newly developed cw-NMR module for polarization detection of solid targets was given, which should serve as a substitute for the Liverpool module. However, possible improvements should not be disregarded. To enable maximum compatibility with existing systems, the new development [4] is based in many ways on the construction of the Liverpool module. Specifically, these are the following:

- exciting RF-signal via external generator

- two demodulation channels

- diode channel for receiving the magnitude spectrum and to tune the NMR resonant circuit

- phase-sensitive detector for the detection of the absorption spectrum

- layout of the connector for power supply and signal transmission

- geometric dimensions of the module

The main differences between the modules can be attributed to various improvements and the necessary replacement of no longer available components:

\footnotetext{
${ }^{3}$ For the operation of a polarized target, this results in the advantage that the necessary dynamic nuclear polarization frequency $\omega_{D N P}$ is kept constant.
} 
- Outsourcing of the resonant circuit components - ensures greater flexibility in experimental set-up of the measuring circuit (e.g. $\lambda / 2$-cable)

- The capacitive elements of the resonant circuit have been replaced by a varicap diode - this allows electronic tuning of the resonant circuit and an access to a measurement based on synchronous resonance tuning (see [5])

- Except the resonant circuit components, all components are on one circuit board with a system-wide impedance of $50 \mathrm{ohms}$ - this allows a simple construction and repair of the modules, also possible signal reflections should be minimized

- Replacement of the radio-frequency amplifier by common models from Mini Circuits - good signal-to-noise ratio and price-performance ratio

- Exchange of rectifier diodes - granted a higher linearity of this channel (see below)

To shield the components against internal and external noise, the boards were placed in specially designed housings of solid brass, which were adjusted to each other.

\section{Test measurements}

For the warranty of functionality of the newly developed cw-NMR module various test measurements were carried out. The most important ones are represented here briefly.

\subsection{Linearity}

A basic requirement for the exact detection of the target polarization over the area units $A$ under the NMR-line is only possible, if the amplification of the signals in the relevant performance
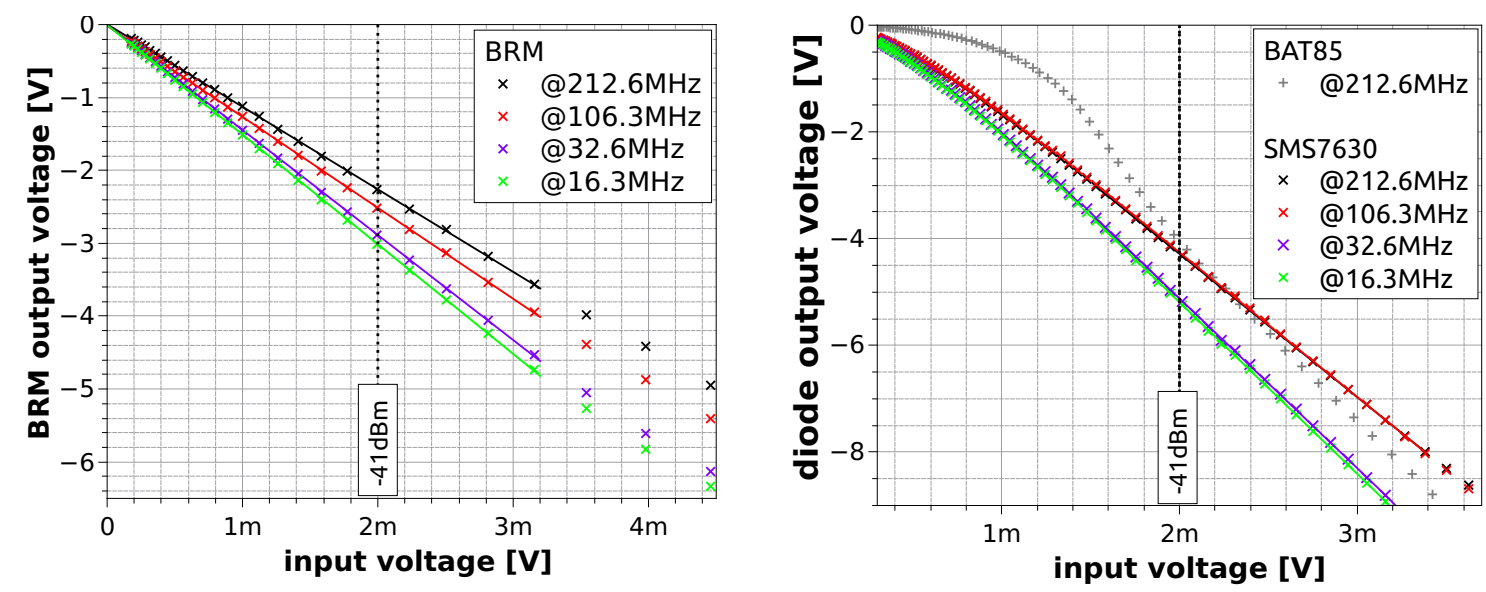

Figure 1: Measuring results for both channels for the observation of the linearity at selected frequencies. As can be seen, a constant gain of the diode channel based on BAT85 diodes was not given. However, by the exchange by SMS7630 diodes, the effect could be eliminated, so that both channels show a high degree of linearity in the measuring range at about $2 \mathrm{mV}$ input voltage $(\hat{=}-41 \mathrm{dBm}$ input power). 

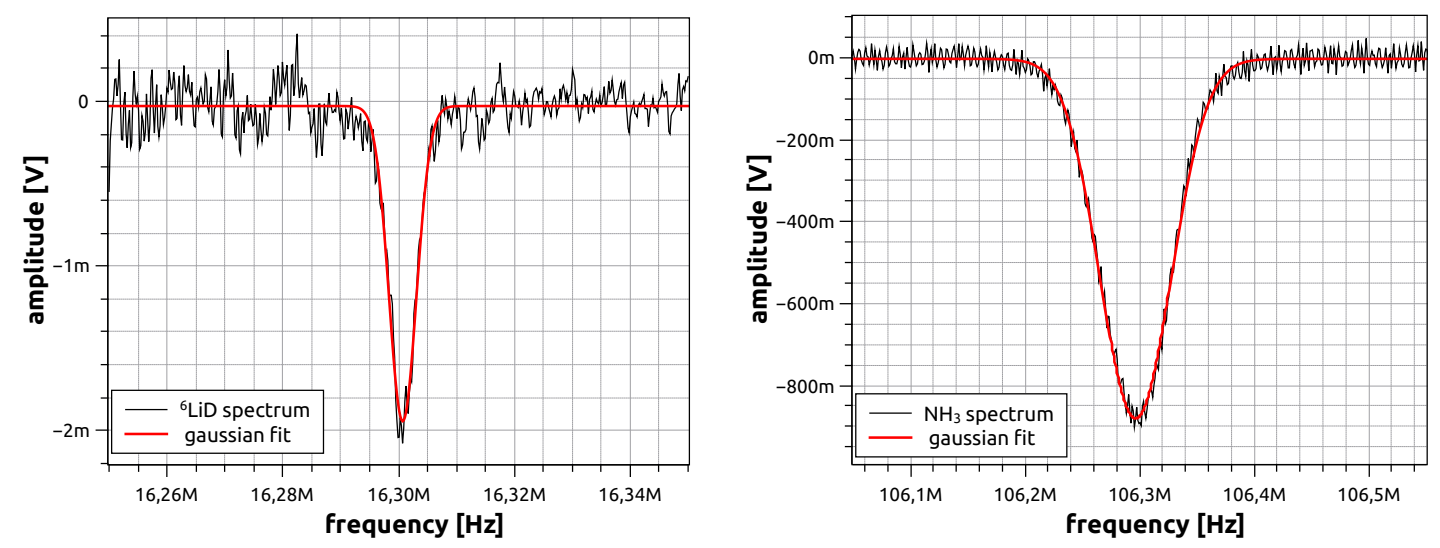

Figure 2: NMR spectra of deuterons $\left({ }^{6} \mathrm{LiD} @ 77 \mathrm{~K} \hat{=} P_{t}=0.0007 \%\right)$ and protons (ammonia @ $1 \mathrm{~K} \hat{=} P_{t}=$ $0.255 \%$ ) in thermal equilibrium at 2.5 Tesla. As can be seen, even smallest signals can be well detected, enabling a sufficiently accurate measurement for a TE-calibration.

range ${ }^{4}$ is constant. Therefore, measurements at different frequencies ${ }^{5}$ were performed to test the linearity of the two channels in this region. The NMR resonant circuit was replaced by a variable attenuator, so that different input power levels $(-62 \mathrm{dBm}$ to $-31 \mathrm{dBm})$ could be simulated.

Considering the resulting voltage profiles of the matched BRM-channel in fig. 1, a high degree of linearity can be seen in the work area for the different frequencies. The varying slopes between these traces at the given frequencies are due to different gains. This fact can be attributed by the frequency dependencies of the used components and is not important for the detection of the polarization at a fixed frequency.

Checking the linearity of the diode-channel based on BAT85-diodes, also used in the Liverpool module, there was a high deviation in a linear amplification at $212.6 \mathrm{MHz}$. This effect could ultimately be attributed only to the diodes used. For this reason, various diodes were tested with respect to their linearity in the work region. It was found that the diode type SMS7630 which, according to data, is especially designed for mixers, shows the most promising behavior. Also measurements in the other relevant frequencies demonstrated the advantages of this type of diode, so that the linearity of this channel could be increased significantly.

\subsection{Detection of real spectra}

In addition to linearity, the possibility for the exact detection of the TE polarization plays an important role. For this reason, the TE signals of proton and deuteron signals of different samples were measured. As can be seen in fig. 2, even smallest signals become clearly apparent from the predominantly white noise of the circuit, so that an exact determination of area units in the thermal equilibrium is possible.

A particular challenge for the polarization detection is represented by the TE signals of quadrupole broadened deuterons, since these spectra are split over a wide frequency range and the correspondingsignal amplitudes are small.

\footnotetext{
${ }^{4}$ The input power of the NMR module during a polarization measurement is, according to experience, at about $-41 \mathrm{dBm}$.

${ }^{5}$ Larmor frequency of deuterons and protons at 2.5 and 5 Tesla.
} 

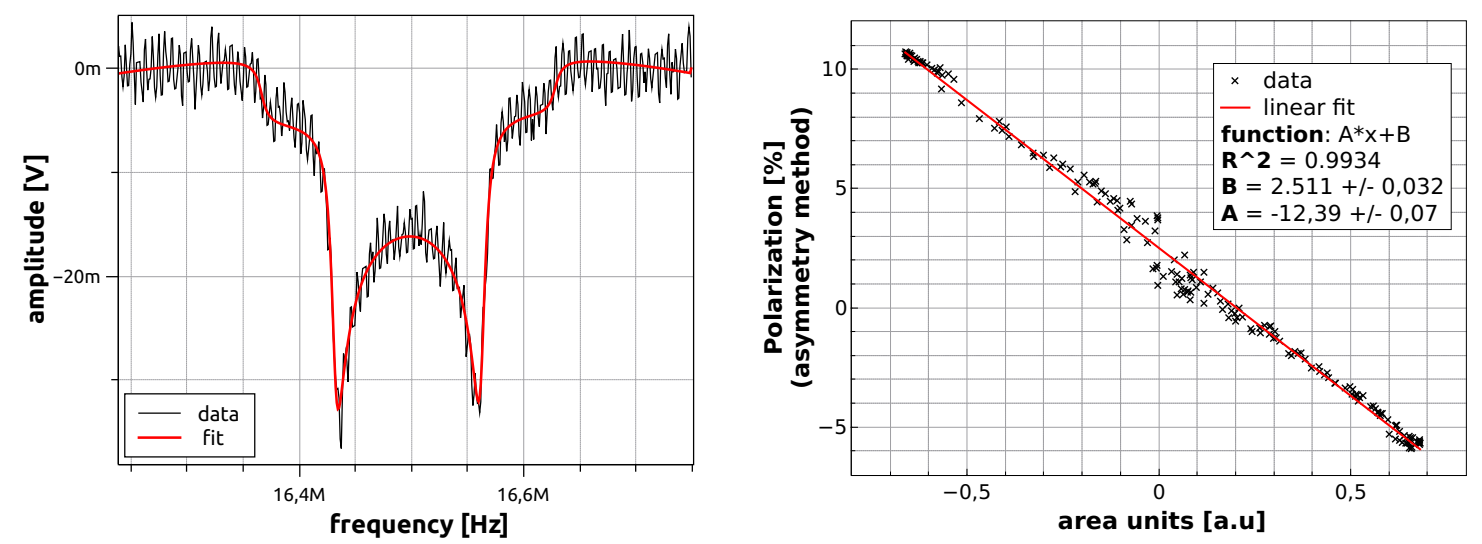

Figure 3: The TE spectra of quadrupole broadened D-butanol and the calculated polarization versus the determined area units of signals. As can be seen, there is an almost linear relationship, thereby providing a high linearity of this channel is illustrated.

Nevertheless, as illustrated by fig. 3, also these spectra can be well detected with the new module, so that a polarization determination based on the TE method is possible.

By the characteristic waveform, which is for example described in [6], a further access to the polarization by the so-called asymmetry method is possible, directly from the line shape. Plotting the determined polarization against the respective area units, as shown in figure 3 , this leads to a linear correlation, where the slope is the known enhancement factor $k$. Comparing the two enhancement factors $\left(k_{T E}=12.22 \pm 0.57\right.$ and $\left.k_{a s y}=12.39 \pm 0.07\right)$ identified for this sample, shows no major contradictions and can thus be used for a confirmation of the correct operation of the module.

\section{Summary}

The various tests have shown that the newly developed cw-NMR-module is ready for experimental use and even smallest signals can be well detected. First measurements were performed at a trityl-doped D-butanol target in Mainz and have shown no discrepancies.

The high compatibility with the Liverpool module also ensures an easy deployment in existing test set-ups, therefore an additional alternative for the polarization detection is available.

\section{References}

[1] F. Bloch, Nuclear Induction, Physical Review, Vol. 70, p. 460-474, 1946.

[2] R.K. Harris, Nuclear Magnetic Resonance Spectroscopy, Longman Scientific \& Technical, 1986.

[3] G.R. Court et al., A high precision Q-meter for the measurement of proton polarisation in polarised targets, NIM A, Vol. 324, p. 433-440, 1993.

[4] J. Herick, Ein neues cw-NMR-Modul und Trityl-dotierte D-Butanol-Targets für Streuexperimente, $\mathrm{PhD}$ thesis, Ruhr-Universität Bochum, 2015.

[5] P. Hautle, Detection of small NMR signals, Proceedings of the Workshop on NMR in Polarized Targets, University of Virginia, 1998.

[6] Ch.M. Dulya et al., A line-shape analysis for spin-1 NMR signals, NIM A, Vol. 398, p. 109-125, 1997. 